\title{
Research and Literature Review on Developing Motion Capture System for Analyzing Athletes Action
}

\author{
HAN Fang ${ }^{1, a}$, BO Xuesong ${ }^{2, b}$ \\ ${ }^{1,2}$ Physical education department of hebei normal university, Shijiazhuang 050024,China \\ ahanfanghb@126.com
}

Keywords: Motion Capture System; Athletes Action; People Matching; Literature Review.

\begin{abstract}
With the development of human-computer interaction technology, pattern, natural interaction will become the main way of interaction between human and computer. Support technology, the computer must understand and capture the human behavior characteristics, so as to put forward the motion capture. The human body is a very complex system, composed of more than 200 rotary joint, if you need to simulate the human body movement of the more vivid, shall provide all joint rotation angle of the value and position of the human body and other relevant information. We conduct literature review on developing motion capture system for analyzing athletes' action in this paper. The result shows the effectiveness of the proposed system. In the future, we plan to do more related experiment to test the robustness of our system.
\end{abstract}

\section{Introduction}

In recent years, the motion capture technology has become a key technique in the study of human movement posture, playing an increasingly important role, people realize the necessary by simulating human movement posture so as to realize the visualization and free 3-d human body skeleton edit control function. At present, many scholars at home and abroad to use existing motion capture techniques to do a lot of research in the field of human motion. In the gaming industry, the market trend is toward active gaming platforms, where a smart wearable motion capture suite could expand the capability of this type of game and open total virtual reality scenarios, at the edge of the augmented reality. Reference [1] using VICON motion capture system to capture human motion data, through the study of the Fourier transform of data processing, and the data after processing applied in robotics research. Literature [2] by using motion capture data in the database for the normal and abnormal motion of the human body is analyzed. Article [3] by using VC + + to build a human motion simulation based on OpenGL technology, flexibility of using acquisition of human motion data driven human body model. These scholars focus on the analysis of human motion data processing and realize the simulation of human motion, but in terms of freedom of motion editing control to the human body and imperfect. Article [4] proposed a novel people matching methodology which will help recognize the body in large degree. More related literatures could be found in [5-8].

Sound system uses the audio signal of flight time to calculate the position of the tag, but the direction of the transmitter/receiver strongly affects the performance of the equipment. In addition, the weakness of magnetic system of management of a limited number of markers and sensitive to external magnetic field is very promising frontier wear-resisting reliable motion capture system is based on the inertial measurement unit (Amos), in almost any place can be used. Relevant business system of the main restriction is the size and weight of a single module. In addition, the existence of the cable connection module is to reduce the abrasion resistance and athletic ability of the system. Business systems, while provides a high level of performance in terms of accuracy, still only a niche market due to their high cost, especially for multiple module system. Many research centers have developed prototype, trying to reduce the limitation of the commercial system in the main, particularly in the size and weight. Based on human motion free edit control imperfect, with VC++ and OpenGL tools, read the motion capture file data, combined with normal human body joint activities, Bessel curve characteristics, and freedom of numerical data, realize the simulation of 
human motion and freedom of the human body movement posture to edit control. More detailed description will be introduced in the following chapters.

\section{The Implementation of the Proposed System}

The Hardware Devices and System Architecture. In this section, the basic idea of the wearable self-contained system capable of recording and reconstructing human motion will be described. Basic aspects correct reconstruction of human movement depends on the biomechanical model of the body, and its accuracy is reflected in the final result. 3-d SUIT is an inertial motion capture system. Mainly includes the following four parts: (1) sensors, mainly used to provide a PC with moving object information; (2) the signal capture equipment, mainly be responsible for the signal capture; (3) data transmission equipment, will capture the data real-time transmission to the PC (3-d SUIT is using a wireless Bluetooth or USB transmission technology); (4) the number of processing equipment, mainly responsible for processing, processing the data collected, including hard data processing equipment (such as PC, etc.) and software (such as Motion-Builder animation software). 3-d SUIT operation process is very simple, and compared with optical motion capture system for environmental requirements are relatively low, only need to wear the clothes equipment on captured objects, connect each sensor to record the serial number of sensors in the human body corresponding position after the real-time motion capture. From a perfect biomechanical model of human body, it may be possible to create a structured suit which was placed on the fixed point. In this way, the reconstruction absolute direction of motion estimation, using the law to move forward. The hardware board and the organization pattern are shown in the figure 1 and 2.
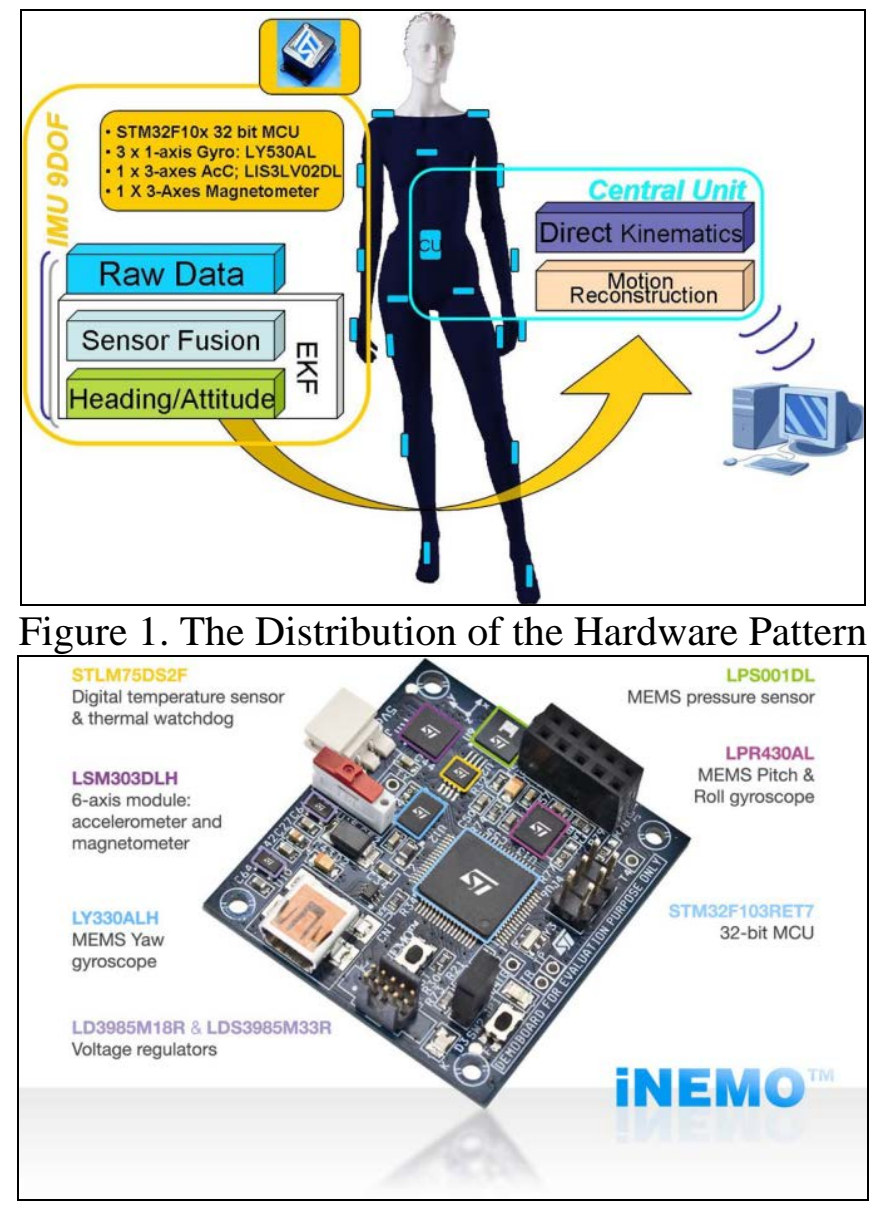

Figure 2. The Adopted Hardware Device

The Human Body Modelling. In the human body skeleton creation stage, create 21 pieces of bone for the body, because every piece of bone containing six data every bone block information contain position information and rotational part two, based on the motion capture data acquisition 
process set the root node Hips as static in the human body, therefore, the location information is static. So when using the program reads the data, only need to rotate the data relative to the parent node. The structure modelling result is shown in the figure 3. Data acquisition is the sensor automatic collection location, rotation Angle and angular velocity of the body. In order to visualize how information that is relevant to human need is to create the skeleton of the human body skin. The skin of the experiment model is fabricated using 3 on3dmax 2009. Bone model is created using the Biped bones, bones and skin through the skin to achieve the correlation between them. In the process of making Skin, respectively to the model using the Physique modifier Skin and Skin compares the modifier, using Skin envelope making process was complicated, and Physique Skin is relatively simple, need to constantly in the process of Skin to make the test, and adjust the parameters of Skin.



Figure 3. The Human Body Modelling

The Mathematical Details for Modelling. The 3-d modelling is expressed as the formula 1 and the initial quaternion conditions are estimated at the start condition by using accelerometer and magnetometer. At the moment, no one has tried to integrate local logging for IMU sensors. This may become a very important feature, particularly in the medical field for long-term experiments with patients. Wireless connectivity would represent a significant improvement on wearability, but power consumption issues and consequently power storage problems.

$$
\left\{\begin{array}{c}
\vec{w}=G_{g} w_{r}+\vec{b}_{w}+\vec{v}_{g} \\
\vec{a}=G_{a}\left[C_{n}^{b}(q)(\vec{g})\right]+\vec{v}_{g} \\
\vec{m}=G_{m}\left[C_{n}^{b}(q)(\vec{m})\right]+\vec{v}_{m}
\end{array}\right.
$$

In the IMUs, the main problem is that the orientation is computed by the integration of the gyro signals, including any superimposed sensor drift and noise. The orientation drift errors, resulting from gyro output errors, can be bounded by additional sensors (accelerometers and magnetometers), whose information allows the correction of the orientation drift. In this way, the Earth's gravitational and magnetic field vectors are resolved by the aiding system in the body frame with their known representation in the North-East-Down (NED) absolute framework.

\section{Experiment and Simulation}

The human body is a very complex system, composed of more than 200 rotary joint, if you need to simulate the human body movement of the more vivid, shall provide all joint rotation angle of the value and position of the human body and other relevant information. Therefore, for simulation of human motion, must first establish a realistic human body skeleton model and then through data 
driving the movement of human body model simulate the motion of human body. Human motion studies is to use motion capture technology, to capture the human body movement, body movement parameters, and reconstruction of the human body structure from motion and posture, realizes the human motion analysis and simulation. According to the research content, this paper chooses a human body interior normal walking and running motion as the input motion. Extract human limbs motion data, simulation of the human arm and leg movement, but as a result of human head and torso motionless, and to simulate the results of limb harmony with motion capture to the human body animation a gap on the lifelike. The result is shown in the figure 4.

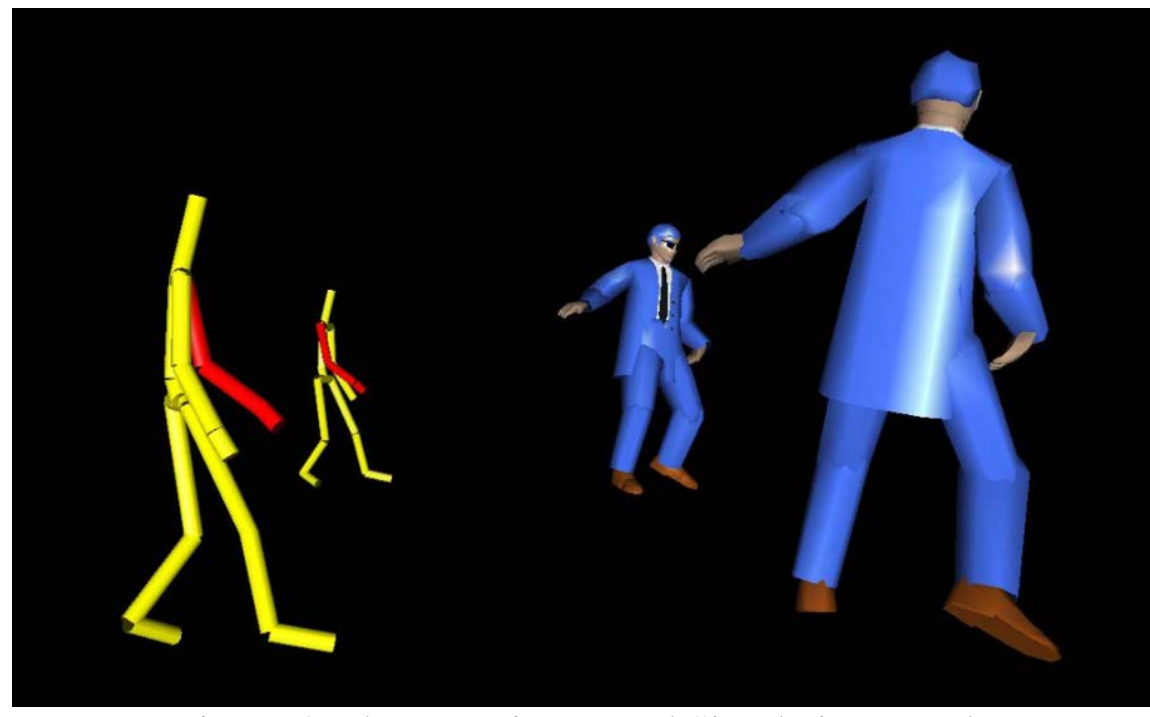

Figure 4. The Experiment and Simulation Result

\section{Conclusion and Summary}

With the development of human-computer interaction technology, pattern, natural interaction will become the main way of interaction between human and computer. Support technology, the computer must understand and capture the human behavior characteristics, so as to put forward the motion capture. Using motion capture techniques, the computer can understand human behavior, and the user can send commands and computer transmit information through the body, scope, the gestures and expressions. Therefore, the motion capture is one of the key technologies of a new generation of human-computer interaction. Modeling, monitoring, and understanding of human motion based on motion capture is a field of research resurgent during the past decade, with the emergence of applications in sports science, medicine, bio-mechanics, animation (online games), monitoring and security. Therefore, we conduct literature review on developing motion capture system for analyzing athletes' action. The experiment simulation result indicates the correctness of proposed method, the accuracy and time-consuming is enhanced significantly.

\section{Acknowledgements}

Social science fund project in hebei province(HB12TTY014).

\section{References}

[1] Michal Vinkler, Jiří Sochor. Integrating Motion Tracking Sensors to Human -Computer Interaction with Respect to Specific User Needs[J]. augmented reality, motion capture, 2014.

[2] Kopniak P. Java wrapper for Xsens motion capture system SDK[C]. //Human System Interactions (HSI), 2014 7th International Conference on. IEEE, 2014:106 - 111. 
[3] Wu J, Wang Z, Raghuraman S, et al. Demonstration abstract: upper body motion capture system using inertial sensors[J]. Information Processing in Sensor Networks, IPSN-14 Proceedings of the 13th International Symposium on, 2014:351 - 352.

[4] Wang H, Shkjezi F, Hoxha E. Distance metric learning for multi-camera people matching[C]. //Advanced Computational Intelligence (ICACI), 2013 Sixth International Conference on. IEEE, 2013:140 - 143.

[5] Monnet T, Samson M, Bernard A, et al. Measurement of three-dimensional hand kinematics during swimming with a motion capture system: a feasibility study[J]. Sports Engineering, 2014, 17(3):171-181.

[6] Park S W, Park H S, Kim J H, et al. 3D displacement measurement model for health monitoring of structures using a motion capture system[J]. Measurement, 2015:352-362.

[7] Kim S, Nussbaum M A. Evaluation of two approaches for aligning data obtained from a motion capture system and an in-shoe pressure measurement system.[J]. Sensors (Basel, Switzerland), 2014.

[8] E C, Z S, C C. Comparison of markerless and marker-based motion capture technologies through simultaneous data collection during gait: proof of concept.[J]. PLoS One, 2014, 9(3):e87640. 